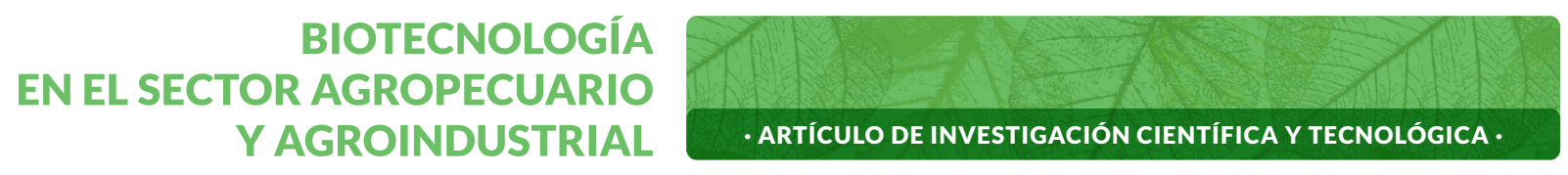

Vol. 19 No 2 · Julio - Diciembre 2021 · ISSN - 1692-3561 · ISSN-e 1909-9959 · https://doi.org/10.18684/bsaa.v19.n2.2021.1425

\title{
Control cinético del salado, con sabor a humo, de filetes de tilapia (Oreochromis sp)*
}

\section{Kinetic control of the smoke-flavoured salting process of tilapia fillets (Oreochromis sp)}

BETANCOURT-SAMBONY, FEDERMAN; RIVERA-VALENCIA, FRANCISCO-JAVIER²;

GIRÓN-HERNÁNDEZ, JOEL ${ }^{3}$

Historial del Artículo

Recibido para evaluación: 17 de Diciembre 2019.

Aprobado para publicación: 11 de Noviembre 2020.

* Título del proyecto de origen: "Desarrollo de un nuevo producto a base de tilapia (Oreochromis sp.) mediante la aplicación de métodos de salado-ahumado al vacío". Financiación: Universidad Surcolombiana. Finalización: 12 de octubre 2019.

1 Universidad Surcolombiana, Facultad de Ingeniería, Dpto de Ingeniería Agrícola. Estudiante de ingeniería Agrícola. Neiva, Colombia. https://orcid.org/0000-0003-1154-6765

2 Universidad Surcolombiana, Facultad de Ingeniería, Dpto de Ingeniería Agrícola. Estudiante de ingeniería Agrícola. Neiva, Colombia. https://orcid.org/0000-0003-4736-7814

3 Universidad Surcolombiana, Facultad de Ingeniería, Dpto de Ingeniería Agrícola. PhD. Ciencia e ingeniería de los alimentos. Neiva, Colombia. https://orcid.org/0000-0003-1245-4475

Correspondencia: joel.giron@usco.edu.co

Cómo citar este artículo: BETANCOURT-SAMBONY, FEDERMAN; RIVERA-VALENCIA, FRANCISCO-JAVIER; GIRÓNHERNÁNDEZ, JOEL. Control cinético del salado, con sabor a humo, de filetes de tilapia (Oreochromis sp). Biotecnología en el sector agropecuario y agroindustrial, v. 19, n. 2, 2021, p. 27-40. Doi: https://doi.org/10.18684/bsaa.v19.n2.2021.1425 


\section{RESUMEN}

La cinética del salado de una matriz muscular expresa el movimiento de la sal mediante modelos difusionales, esto permite predecir el resultado final del producto procesado. Este trabajo estudió el salado de filetes de tilapia utilizando $\mathrm{NaCl}$ y sal ahumada, con envasado al aire y al vacío. Se midieron los cambios de masa, de agua y de sal en los filetes después del salado. Adicionalmente, se les realizó la determinación de $\mathrm{pH}$, color, textura, $a_{w}$, humedad, grasa y cloruros. La información se procesó mediante: i) modelos matemáticos para determinar la cinética de la sal, ii) ANOVA multifactorial para observar el efecto de los factores en las variables respuesta y iii) la metodología de superficie de respuesta para optimizar el proceso. Los resultados obtenidos mostraron un transporte pseudo-difusional de la sal. En el ANOVA multifactorial, el factor tiempo presentó la mayor influencia en las variables respuesta. Finalmente, la modelización del proceso determinó que el mejor salado se obtiene a los 161 minutos para conseguir una $a_{w}$ de 0,95 y un contenido de sal en base seca de 0,17 g empleando sal ahumada y el envasado al vacío. El producto obtenido cumplió lo establecido por el Codex Alimentarius para pescado salado.

\section{ABSTRACT}

The kinetics of salting a muscular matrix expresses the movement of the salt by means of diffusion models, allowing the prediction of the result of the processed product. This work studied the salting of tilapia fillets using $\mathrm{NaCl}$ and smoked salt, with air and vacuum packaging. The changes in mass, water and salt in the fillets after salting were measured. In addition, the fillets were investigated for $\mathrm{pH}$, colour, texture, aw, moisture, fat, and chlorides. The information was processed using: i) mathematical models to determine the kinetics of salt, ii) multifactorial ANOVA to observe the effect of factors on response variables and iii) response surface methodology to optimise the process. The results obtained showed a pseudo-diffusional transport of salt. In the multifactor ANOVA, the time factor had the greatest influence on the response variables. Finally, the modelling of the process determined that the best salt was obtained after 161 minutes to achieve an aw of 0,95 and a dry-base salt content of $0,17 \mathrm{~g}$ using smoked salt and vacuum packaging. The product obtained complied with the requirements of the Codex Alimentarius for salted fish.

\section{INTRODUCCIÓN}

En Colombia el cultivo de especies acuáticas se remonta a la década de los años 30 cuando se importó la trucha arco iris (Oncorhynchus mykiss) con el objetivo de repoblar aguas de uso público para la pesca deportiva (Parrado-Sanabria, 2012). Posteriormente, entre 1959 y 1967 se introdujo la tilapia (Oreochromis sp.) con fines comerciales. Sin embargo, hasta principio de los años noventa con las exportaciones de filetes a los Estados Unidos (Rodríguez-Ortigoza, 2017) se dio el auge del sector. El Departamento del Huila es en la

\section{PALABRAS-CLAVE:}

Cinética; Color; Difusión de sal ahumada; Filete; Modelización; Salado al vacío; Sal ahumada; Metodología de superficie de respuesta; Tilapia; Textura.

\section{KEYWORDS:}

Kinetics; Colour; Smoked salt diffusion; Fillet; Modelling; Vacuum salting; Smoked salt; Response surface methodology; Tilapia; Texture. 
actualidad el primer productor piscícola con el 44,46 \% de la producción nacional, seguido por Meta con el $14,07 \%$ y Tolima con el 4,87 \% (Mariño-García, 2018). Buena parte de la tilapia generada en el Huila se dedica a la exportación y se comercializa principalmente en forma de filetes frescos, filetes congelados y tilapia entera congelada (García-Bernal, 2019). En caso de no poderse exportar el producto se generaría un impacto económicamente considerable sobre las empresas piscícolas al ser este un alimento de baja demanda en el mercado nacional (Camero-Escobar and Calderón-Calderón, 2018). La diversidad de productos derivados de la tilapia no suele ser muy abundante; hasta el momento, no hay una producción diferente a la de hamburguesas, salchichas y lomitos. Pocos son los procesos de transformación que se desarrollan a base de pescado, y no hay mayores iniciativas debido a las características del producto. La innovación a pequeña escala puede generar mejoras significativas para la industria pesquera, siempre y cuando el nuevo producto se adapte a los requisitos del cliente sin dejar de ser eficiente en términos del tiempo de entrega (Fouad et al., 2018). Los platos preparados están ganando popularidad en los mercados minoristas y en la alimentación con entrega a domicilio debido que ahorran tiempo y están listos ser consumidos y tienen una segmentación de mercado bien definida (Guimarães et al., 2019).

El salado es un método utilizado en la transformación y conservación de pescado, se desarrolla en salmuera o en seco (Laub-Ekgreen and Flemming, 2019). El método en seco consiste en aplicar directamente la sal sobre el músculo y se recomienda en especies de pescados con bajo contenido graso (menor al $8 \%$ ). Por otro lado, el salado en salmuera suele realizarse en especies con contenidos grasos por encima del $8 \%$. La tilapia presenta un contenido graso que suele estar por debajo de 7 \% (García et al., 2017), lo que la hace apta para realizar un salado en seco.

Los procedimientos de salado se han ido modificando con el objetivo de reducir los tiempos, para esto se han aplicado alternativas como el envasado al vacío (Martins et al., 2019) que genera un salado más homogéneo como consecuencia de la distribución de la sal en el producto, favoreciendo la transferencia de masa gracias a que el vacío mejora la superficie de contacto debido a la eliminación del aire dentro de los poros sin afectar los parámetros fisicoquímicos, (Deumier et al., 2004) y además, se disminuye la producción de residuos (Fuentes et al., 2008). Otros estudios se han centrado en la calidad organoléptica del producto al adicionar sabor a humo, y han evaluado la influencia de propiedades como: la matriz del pescado, los procedimientos de salado, el tipo de humo, el tiempo de ahumado, la temperatura y la humedad relativa (Rizo et al., 2017a), lo que confiere al producto final características con una buena aceptación sensorial. Por tanto, en este trabajo se estudió el control cinético del salado en seco para conocer los procesos difusionales al utilizar $\mathrm{NaCl}$ y sal ahumada en la matriz muscular, y así encontrar la combinación de factores que permitieran obtener la concentración de sal ideal en los filetes de tilapia.

\section{MÉTODO}

En el estudio se utilizaron un total de 88 filetes de tilapia con un peso promedio de $226 \pm 44,3 \mathrm{~g}$, que se obtuvieron de una comercializadora en el municipio de Aipe ubicado a una altitud de 350 m.s.n.m. en el departamento del Huila $\left(3^{\circ} 13^{\prime} 19^{\prime \prime} \mathrm{N}, 75^{\circ} 14^{\prime} 15^{\prime \prime} \mathrm{O}\right)$, con promedios anuales de $28^{\circ} \mathrm{C}$ temperatura y $66,5 \%$ humedad relativa (IDEAM). La Figura 1 muestra el esquema de la metodología seguida, la caracterización de la materia prima y los procedimientos de salado realizados en el laboratorio de análisis de alimentos del grupo de Agroindustria de la Universidad Surcolombiana.

\section{Preparación y salado de las muestras}

El experimento se inició con la determinación de las características fisicoquímicas de la materia prima ( $\mathrm{n}=8$ ); para esto se realizaron las determinaciones de humedad en base húmeda $\left(\mathrm{x}^{\mathrm{w}}\right), \mathrm{pH}$, textura, color $\left(\mathrm{L}^{*}, \mathrm{a}^{*}, \mathrm{~b}^{*}\right)$, contenido de grasas $\left(x^{f}\right)$ y la $a_{w^{*}}$. Posteriormente, se realizó el salado de los filetes siguiendo el procedimiento adaptado de (Rizo et al., 2017b), se pesaron los filetes de tilapia $(n=80)$ y se hizo la dosificación de sales $\left(x^{\mathrm{NaCl}}=0,26 \% \mathrm{p} / \mathrm{p}\right) \operatorname{con~NaCl}$ $(n=40)$ y sal ahumada $(n=40)$, los cuales fueron envasados al aire $(n=20)$ y al vacío $(n=20)$, para los dos tipos de sal; para la evaluación de cada tiempo de salado se emplearon dos filetes. Después del salado, se establecieron diez 
tiempos de muestreo, desde 33 hasta 330 minutos y se realizaron las determinaciones fisicoquímicas mencionadas a cada tratamiento. Adicionalmente, se midieron los contenidos de cloruros en base húmeda ( $\left.x^{\mathrm{NaCl}}\right)$, en base seca $\left(\mathrm{X}^{\mathrm{NaCl}}\right)$, en la fase líquida ( $\left.Z^{\mathrm{NaCl}}\right)$ y las variaciones de: masa total $\left(\Delta \mathrm{M}_{\mathrm{o}}\right)$, masa de agua $\left(\Delta \mathrm{M}_{\mathrm{w}}\right)$ masa de sal $(\Delta \mathrm{NaCl})$.

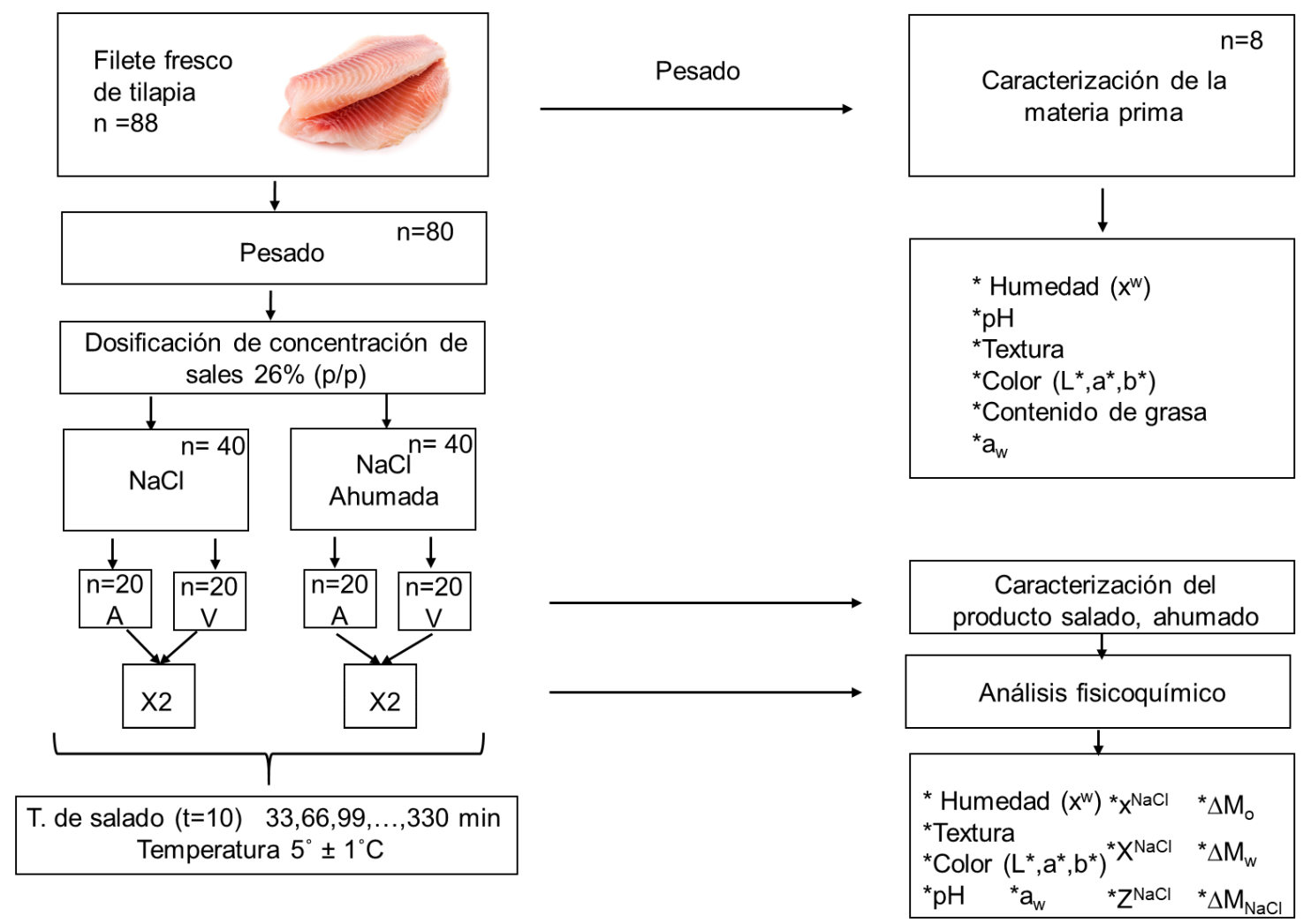

Figura 1. Esquema de la metodología realizada para evaluar y determinar la cinética del salado, con sabor a humo, de filetes de tilapia a condiciones de salado al aire $(\mathrm{A})$ y a vacío (V).

\section{Determinaciones analíticas}

Contenido de Humedad. El contenido de humedad se determinó mediante un horno de secado UF30plus (Memmet, Alemania) siguiendo el método propuesto por la AOAC, 1997. El porcentaje de humedad expresado en $\mathrm{g}$ de agua por $100 \mathrm{~g}$ de muestra se obtuvo usando la ecuación 1 y posteriormente se calculó la humedad en base seca empleando la ecuación 2.

$$
\begin{aligned}
& \% x^{w}=\frac{\left(p_{1}-p_{2}\right)}{\left(p_{1}\right)} \times 100 \\
& \% X^{w}=\frac{\left(p_{1}-p_{2}\right)}{\left(p_{2}\right)} \times 100
\end{aligned}
$$

Donde:

$P_{1}$ : Peso inicial de la muestra en $g$

$P_{2}$ : Peso final de la muestra en $g$ 
Contenido de Cloruro sódico. El contenido en cloruro sódico ( $\left.X^{\mathrm{NaCl}}\right)$ se estableció homogeneizando $1 \mathrm{~g}$ de la muestra triturada en agua destilada, siguiendo el procedimiento establecido por Girón et al. (2015) mediante un analizador automático de cloruros Sherwood 926S (Sherwood Scientific Ltd., UK). A partir de la lectura que arroja el equipo ( $\mathrm{mg}$ de ion cloruro/L) se calculó la concentración de cloruro sódico en las muestras mediante la ecuación 3.

$$
\frac{\mathrm{g} \mathrm{NaCl}}{100 \mathrm{~g} \text { muestra }}=\frac{\text { lectura } \times \mathrm{V} \times 58,5}{p \times 35,5 \times 10}
$$

Donde:

Lectura: Valor dado por el equipo (mg Cl/L)

$\mathrm{V}$ : Volumen de la disolución de pescado (L)

p: Masa de la muestra (g)

La concentración de $\mathrm{NaCl}$ en fase líquida ( $Z^{\mathrm{NaCl}}$ ) y el contenido de sal en base seca ( $X^{\mathrm{NaCl}}$ ) se calcularon a partir de los valores de humedad $\left(X^{\mathfrak{w}}\right)$ y contenido en $\mathrm{NaCl}\left(X^{\mathrm{NaCl}}\right)$ según las ecuaciones 4 y 5 .

$$
\begin{gathered}
Z^{\mathrm{NaCl}}=\frac{x^{\mathrm{NaCl}}}{x^{w}+x^{\mathrm{NaCl}}} \\
X^{\mathrm{NaCl}}=\frac{x^{\mathrm{NaCl}}}{1-x^{w}}
\end{gathered}
$$

Bases de cálculo. Para evaluar las variaciones de masa total, agua y cloruro sódico durante el proceso de salado se utilizaron las bases de cálculo según las ecuaciones 6, 7 y 8.

Variación de masa total:

$$
\Delta M_{t}^{o}=\left(\frac{M_{t}^{o}-M_{0}^{o}}{M_{0}^{o}}\right)
$$

Variación de masa de agua:

$$
\Delta M_{t}^{w}=\left(\frac{M_{t}^{o} \cdot x_{t}^{w}-M_{0}^{o} \cdot x_{0}^{w}}{M_{0}^{o}}\right)
$$

Variación de masa de cloruro sódico:

$$
\Delta M_{t}^{\mathrm{NaCl}}=\left(\frac{M_{t}^{o} \cdot x_{t}^{\mathrm{NaCl}}-M_{0}^{o} \cdot x_{0}^{\mathrm{NaCl}}}{M_{0}^{o}}\right)
$$


Donde:

$M_{t}^{o}:$ Masa de la muestra a tiempo t $(\mathrm{g})$

$M_{0}^{o}$ : Masa inicial de la muestra $(\mathrm{g})$

$x_{t}^{w}$ : Fracción másica de agua a tiempo $\mathrm{t}$ (g O/g pescado)

$x_{0}^{w}$ : Fracción másica de agua inicial $\left(\mathrm{g} \mathrm{H}_{2} \mathrm{O} / \mathrm{g}\right.$ pescado)

$x_{t}^{N a C l}$ : Fracción másica de cloruro sódico a tiempo $\mathrm{t}$ ( $\mathrm{g} \mathrm{NaCl} / \mathrm{g}$ pescado)

$x_{0}^{\mathrm{NaCl}}$ : Fracción másica de cloruro sódico inicial (g NaCl/g pescado)

Contenido de grasas. El contenido de grasa se determinó por extracción en Soxhlet mediante un extractor de grasas Soxtec ST 255 TM (Foss, Dinamarca) con éter de petróleo según el método 991.36 (AOAC, 1997). El porcentaje de grasa en las muestras de tilapia se calculó mediante la ecuación 9:

$$
\% \text { grasa }=\frac{\left(m_{2}-m_{1}\right)}{\left(m_{0}\right)} \times 100
$$

Donde:

$\mathrm{m}_{0}$ : Masa de la muestra $(\mathrm{g})$

$\mathrm{m}_{1}$ : Masa del matraz $(\mathrm{g})$

$\mathrm{m}_{2}$ : Masa del matraz con la grasa extraída (g)

pH. El pH de las muestras se midió a partir del pescado homogeneizado en agua destilada (1:10 p/v), haciendo uso de un pH metro BP3001 (Trans Instruments, Singapur), con un electrodo combinado con sensor de temperatura incorporado.

Actividad de agua $\left(a_{w}\right)$. Las medidas de $a_{w}$ se realizaron a partir de un higrómetro de punto de rocío AquaLab ${ }$ CX-2 (Decagon Devices Inc., EE. UU).

Determinación de color. La determinación del color se realizó directamente sobre los filetes; se utilizó un colorímetro móvil CR-410 HEAD (Konica Minolta, Japón), utilizando el iluminante D65 y el observador estándar de $10^{\circ}$. Se tuvieron como referencia las coordenadas colorimétricas del sistema CIE L* $a^{*} b^{*}$ (1976). Las medidas se realizaron después del salado en tres puntos diferentes, aproximadamente a tres centímetros de los bordes del filete.

Textura. La textura de los filetes de tilapia, frescos y procesados se determinó con un analizador de textura CT3 (Brookfield Eng. Lab., EE. UU). Se aplicó una prueba APT, la muestra se conformó por bloques de la matriz de 10 x $10 \times 10 \mathrm{~mm}$, tomados de 2 filetes de pescado, sobre los cuales se aplicó doble compresión, con celda de carga de $10 \mathrm{~kg}$ y émbolo cilíndrico plano (TA4/100) con un espesor de $20 \mathrm{~mm}$ y diámetro de $38 \mathrm{~mm}$; se utilizó una velocidad de 0,8 mm/s con una carga de activación de 0,15 N y un 50 \% de deformación.

\section{Diseño experimental}

Se realizó un diseño experimental completamente al azar con un arreglo factorial $10^{*} 2 * 2$, para un total de cuarenta combinaciones resultantes de tres factores: i) tiempo (T) con diez niveles de salado (33 a $330 \mathrm{~min}$ ), ii) envasado (E) dos condiciones (aire y vacío), iii) sal (S) dos clases ( $\mathrm{NaCl}$ y $\mathrm{NaCl}$ ahumada) 
Análisis estadístico. Con la información adquirida de la variación total de masa, de agua y de $\mathrm{NaCl}$ se observó el comportamiento cinético de la sal en la matriz muscular; mediante el modelo mencionado por (Chang-Cheng and Jong-Bang, 2018a), ecuación 10.

$$
\Delta M_{t}^{i}=\left(1+1 k_{1}+k_{1} * t^{0,5}\right)
$$

Donde $t$ es el tiempo de salmuera (h), y $k_{1} y k_{2}$ son los coeficientes de difusión.

Seguidamente, se utilizaron diferentes modelos estadísticos de regresiones y se seleccionó el de mejor ajuste, verificando que los datos utilizados cumplían las condiciones de normalidad, homocedasticidad e independencia. Posteriormente, se aplicó un ANOVA multifactorial con los valores de las determinaciones fisicoquímicas (variables respuesta) con el objetivo de evaluar el efecto de los factores: tiempo de salado, condición de envasado, tipo de sal y sus interacciones. Finalmente, se empleó la Metodología de la Superficie de Respuesta (RSM) para establecer la combinación de los factores que maximizan el rendimiento del salado. Los procedimientos se realizaron con las licencias de los programas Microsoft Excel 2016 (Microsoft Corporation, EE. UU)., y Statgraphics Centurion XVI (Manugistics. USA).

\section{RESULTADOS}

\section{Caracterización de la materia prima}

El cuadro 1 presenta la composición fisicoquímica del filete de tilapia fresco, los datos se expresan en valores promedio y desviación estándar, indicando que la caracterización de la materia prima coincidió con lo reportado por otros autores (Oliveira-Alcântara et al., 2019; Monteiro et al., 2019).

Cuadro 1. Caracterización fisicoquímica de los filetes crudos de tilapia, valores promedio \pm desviación estándar.

\begin{tabular}{|l|c|}
\hline \multicolumn{1}{|c|}{ Determinación analítica } & Tilapia fresca \\
\hline Humedad $\left(\mathrm{x}^{\mathrm{w}}\right)$ & $70,34 \pm 1,34$ \\
\hline Grasa $\left(\mathrm{x}^{\mathrm{g}}\right)$ & $2,78 \pm 0,05$ \\
\hline $\mathrm{pH}$ & $6,21 \pm 0,32$ \\
\hline $\mathrm{a}_{\mathrm{w}}$ & $0,98 \pm 0,01$ \\
\hline $\mathrm{L}^{*}$ & $57,15 \pm 5,32$ \\
\hline $\mathrm{a}^{*}$ & $7,88 \pm 1,13$ \\
\hline $\mathrm{b}^{*}$ & $6,71 \pm 0,71$ \\
\hline Dureza $(\mathrm{N})$ & $54,57 \pm 7,80$ \\
\hline Adhesividad $(\mathrm{mJ})$ & $2,75 \pm 1,17$ \\
\hline Cohesividad & $0,35 \pm 0,04$ \\
\hline Elasticidad $(\mathrm{mm})$ & $4,24 \pm 0,88$ \\
\hline Firmeza $(\mathrm{N})$ & $11,67 \pm 2,13$ \\
\hline Masticabilidad $(\mathrm{mJ})$ & $39,19 \pm 7,99$ \\
\hline
\end{tabular}




\section{Caracterización del salado}

La Figura 2 muestra el mejor ajuste de los datos, obtenido mediante modelos de regresiones lineales, para la evolución de: la variación de masa total, la variación de masa de agua y la variación de masa de cloruro de sodio para los filetes de tilapia salados con los dos tipos de sal y envasados al aire y al vacío.

Variaciones de masa total. En todos los tratamientos las mayores pérdidas de masa se dieron en los primeros tiempos de salado y a medida que aumentaba el tiempo disminuía la pérdida de masa total, Figura 2(A), asumiéndose un transporte pseudo-difusional debido a la dependencia respecto al tiempo (Chang-Cheng and Jong-Bang, 2018b). Este comportamiento se hizo presente en los dos tipos de sal y envasado, las mayores variaciones de masa se evidenciaron al aplicar $\mathrm{NaCl}$ con envasado al vacío. Todos los modelos obtenidos presentaron valores de coeficientes de determinación superiores al 0,91 para todos los tratamientos; en todos los casos, la masa del filete disminuyó hasta llegar a un punto de equilibrio entre la concentración del medio salino y la matriz alimentaria (Casales and Yeannes, 2016; López-Valencia, 2017). Se observó que con $\mathrm{NaCl}$ y envasado al vacío la velocidad de penetración de la sal en el músculo fue la mayor $\left(0,1465 \mathrm{~s}^{0,5}\right)$ de todos los tratamientos; el resultado se debe a que la aplicación de vacío hace que aumente transferencia de masa como consecuencia de la eliminación del aire dentro de los poros, lo que permitió la interacción entre las partículas de $\mathrm{NaCl}$ y la superficie de contacto del músculo; mientras que al utilizar $\mathrm{NaCl}$ y el envasado al aire la velocidad del proceso fue de 0,0294 $\mathrm{s}^{0,5}$, siendo la menor de todos los métodos evaluados, lo que se debe a la desnaturalización del músculo que genera encostramiento de la superficie de la matriz (Petit et al., 2019); por tanto, se ralentizó la penetración de la sal en la matriz. Al analizar la variación de masa total, en los tratamientos realizados con sal ahumada, no se observaron diferencias al utilizar envasado al aire o al vacío con valores de $0,0524 \mathrm{~s}^{0,5}$ y $0,0628 \mathrm{~s}^{0,5}$ respectivamente.

Variaciones de masa de agua. Se exhibieron pérdidas graduales del contenido de agua a medida que aumentó el tiempo de salado en los métodos evaluados, Figura 2(B). Adicionalmente, durante el proceso de salado hubo una difusión del $\mathrm{NaCl}$ y del azúcar (presente en la mezcla de sal ahumada) hacia el músculo del pescado, mientras que el agua se movilizó hacia los medios circundantes para diluir los solutos como consecuencia de los mecanismos osmóticos y de difusión (Tirado-Armesto et al., 2016; Galvão-Martins et al., 2019). Al comparar los tratamientos evaluados, la mayor pérdida de agua se presentó al utilizar $\mathrm{NaCl}$; el vacío aceleró las pérdidas para los dos tipos de sal utilizados.

Variaciones de masa de $\mathrm{NaCl}$. En todos los tratamientos evaluados se observó un incremento de los sólidos solubles medidos en fracción de cloruro de sodio en función del tiempo de procesado; los resultados concuerdan con lo propuesto por otros autores (Erkan, 2017). Los filetes salados con $\mathrm{NaCl}$ exhibieron mayor ganancia de cloruros en comparación con el salado con sal ahumada. El NaCl produjo una deshidratación acelerada del músculo con velocidades de $0,011 \mathrm{~s}^{0,5}$ para el salado al aire y $0,0109 \mathrm{~s}^{0,5}$ para el salado al vacío, lo que afectó el contenido de agua y como consecuencia la masa de los filetes; tal y como se observó en las variaciones de masa total y masa de agua.

La sal ahumada, debido a su composición, azúcares $50 \%, \mathrm{NaCl} 49 \%$ y grasas $1 \%$, evidenció que la incorporación de solutos fue más lenta y uniforme en comparación con los tratamientos realizados con $\mathrm{NaCl}$. En los filetes procesados con envasado al vacío, al restablecer la presión atmosférica, se generó la impregnación de los capilares del tejido, debido a que los poros se llenaron con la solución formada entre el cloruro de sodio y el agua del filete; como resultado disminuyó la actividad de agua del tejido (Bjørnevik et al., 2018). Adicionalmente, en este procedimiento se aportó al mismo tiempo el sabor a humo.

El cuadro 2 exhibe los resultados del ANOVA multifactorial para cada uno de los parámetros evaluados durante el proceso de salado. Tanto el tiempo de salado, el envasado y las interacciones de (TxE) y (ExS) influenciaron las variables de humedad en base húmeda y humedad en base seca, lo que se refleja en los menores contenidos de humedad en los filetes al final del proceso (Figura 2B). Adicionalmente, la interacción (TXS) únicamente presentó influencia en la variable de humedad en base seca, como se observa el contenido de solutos presentes en de la matriz (Figura 2C). 


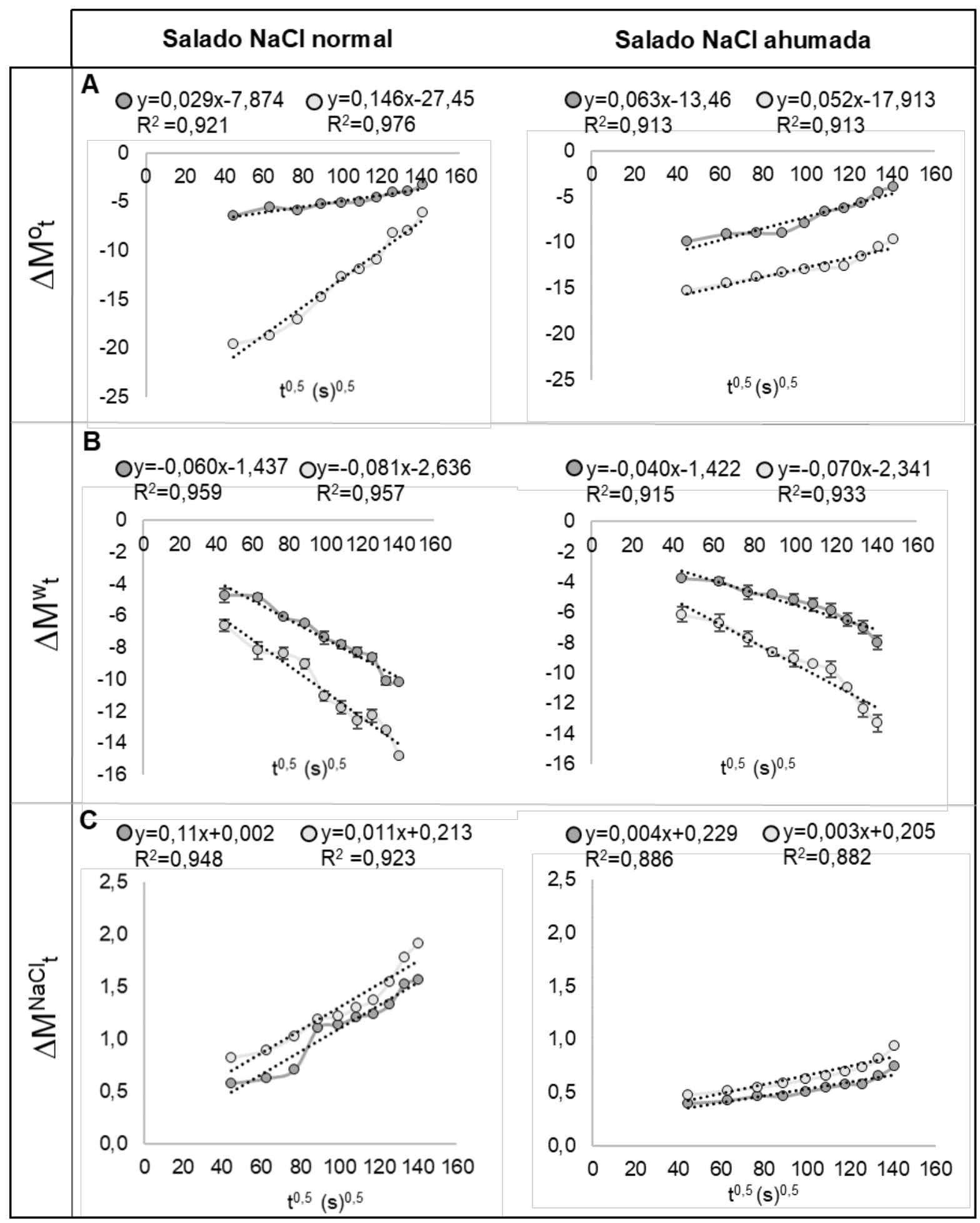

Figura 2. Evolución de la variación de masa total (A), la variación de masa de agua (B) y la variación de masa de cloruro de sodio (C) para los filetes de tilapia salados con los dos tipos de sal y envasados al aire y al vacío 
Cuadro 2. Valores de la razón F y los niveles de significancia obtenidos en ANOVA multifactorial para las variables respuesta según los factores: tiempo (T), condiciones de envasado (E), tipo de sal (S) y sus interacciones (TxE, TxS, ExS).

\begin{tabular}{|c|c|c|c|c|c|c|}
\hline & $\mathbf{T}$ & $\mathbf{E}$ & $S$ & TxE & TxS & ExS \\
\hline $\mathrm{x}^{\mathrm{w}} \mathrm{Bh}(\%)$ & $48,4^{* * *}$ & $112,2^{* * *}$ & $0,1^{\text {ns }}$ & $3,1^{* *}$ & $1,5^{\text {ns }}$ & $14,0^{* * *}$ \\
\hline $\mathrm{X}^{\mathrm{w}} \mathrm{Bs}(\%)$ & $62,8^{* * *}$ & $147,8^{* * *}$ & $0,1^{\text {ns }}$ & $2,9^{* *}$ & $2,1^{*}$ & $17,4^{* * *}$ \\
\hline $\mathrm{pH}$ & $101,5^{* * *}$ & $3,8^{\text {ns }}$ & $8,8^{* * *}$ & $17,2^{* * *}$ & $11,1^{* * *}$ & $9,6^{* * *}$ \\
\hline Dureza (N) & $436,8^{* * *}$ & $3722,4^{* * *}$ & $669,7^{* * *}$ & $29,4^{* * *}$ & $27,8^{* * *}$ & $157,6^{* * *}$ \\
\hline Adhesividad (mJ) & $318,9^{* * *}$ & $688,6^{* * *}$ & $749,5^{* * *}$ & $58,8^{* * *}$ & $16,8^{* * *}$ & $118,7^{* * *}$ \\
\hline Cohesividad & $321,2^{* * *}$ & $105,4^{* * *}$ & $441,5^{* * *}$ & $1,4^{\mathrm{ns}}$ & $2,4^{*}$ & $0,7^{\text {ns }}$ \\
\hline Elasticidad (mm) & $148,9^{* * *}$ & $775,1^{* * *}$ & $620,6^{* * *}$ & $12,2^{* * *}$ & $6,5^{* * *}$ & $5,4^{*}$ \\
\hline Firmeza (N) & $310,5^{* * *}$ & $3097,1^{* * *}$ & $11,1^{* *}$ & $24,8^{* * *}$ & $1,3^{\text {ns }}$ & $643,9^{* * *}$ \\
\hline Masticabilidad (mJ) & $339,0^{* * *}$ & $2985,8^{* * *}$ & $97,4^{* * *}$ & $83,9^{* * *}$ & $6,6^{* * *}$ & $327,9^{* * *}$ \\
\hline \multicolumn{7}{|c|}{ Coordenadas de color } \\
\hline$L^{*}$ & $43,6^{* * *}$ & $116,0^{* * *}$ & $41,1^{* * *}$ & $13,6^{* * *}$ & $20,1^{* * *}$ & $481,7^{* * *}$ \\
\hline$a^{*}$ & $10,8^{* * *}$ & $13,4^{* * *}$ & $619,7^{* * *}$ & $10,1^{* * *}$ & $13,7^{* * *}$ & $4,4^{*}$ \\
\hline$b^{*}$ & $26,7^{* * *}$ & $79,1^{* * *}$ & $766,2^{* * *}$ & $28,8^{* * *}$ & $32,1^{* * *}$ & $0,4^{\text {ns }}$ \\
\hline
\end{tabular}

En la determinación de textura, los factores evaluados y sus interacciones mostraron efecto en los parámetros de: dureza, adhesividad, elasticidad y masticabilidad. Para la cohesividad y la firmeza, todos los factores en estudio presentaron influencia; sin embargo, las interacciones (TxE) y (ExS) no afectaron la cohesividad y (TxS) no influyó en la firmeza.

Diferentes autores encontraron que durante el proceso del salado en pescado la estructura de la matriz muscular del filete evidencia la desnaturalización y solubilidad de las proteínas lo que genera el ablandamiento de las fibras musculares (Bouletis et al., 2017; Jiang et al., 2019). Se observó la disminución en los valores de todos los parámetros de textura con el aumento del tiempo. Adicionalmente, en los tratamientos en los que se aplicó sal ahumada la disminución fue menos pronunciada en comparación con los tratamientos en los que se aplicó $\mathrm{NaCl}$.

En relación con las coordenadas de color se evidenció que tanto los factores y sus interacciones presentaron efecto durante el salado, a excepción de la coordenada amarillo-azul ( $b^{*}$ ) en la que la interacción (ExS) no fue influyente. Para la coordenada de luminosidad $\left({ }^{*}\right)$ se observó una disminución en todos los tratamientos con respecto al tiempo, esto se atribuye a la pérdida de agua producida durante el almacenamiento de las muestras saladas al aire, mientras que, para las coordenadas $\mathrm{a}^{*}$ y b* se observó una disminución en los valores a medida que transcurrió el tiempo, cuando el salado se realizó con $\mathrm{NaCl}$; caso contrario al de los filetes salados con sal ahumada, en el que el valor de las coordenadas aumentó debido a pigmentos provenientes del humo. En general, para todas las determinaciones fisicoquímicas los factores fueron más influyentes de manera individual que sus interacciones.

\section{Modelización y maximización del proceso de salado}

El cuadro 3 presenta los coeficientes resultantes de la RSM para el salado con sal ahumada y $\mathrm{NaCl}$ obtenidos a partir de los factores tiempo (minutos), tipo de sal y tipo de envasado con sus respectivas interacciones para las variables de actividad de agua, contenido de cloruro de sodio en fase líquida y contenido de cloruro de sodio en base seca, con el fin de calcular el contenido de sal adecuado en el salado de filetes de tilapia. Para el tratamiento de $\mathrm{NaCl}$ no se muestran los coeficientes para el tipo de sal ni para su respectiva interacción debido a que la sal ahumada resultó como factor ideal. Se encontró que los $\mathrm{R}^{2}$ y los $\mathrm{R}^{2}$ ajustados estaban por encima del $80 \%$ para las variables de referencia, los valores de estos coeficientes fueron mayores para los filetes tratados con sal ahumada y con envasado al vacío frente a los tratamientos realizados con $\mathrm{NaCl}$ y envasado al vacío. Por lo anterior, se simplificó el diseño experimental a un arreglo factorial $10^{*} 2$, en el que se suprimió el tipo de sal. Las condiciones de tiempo y envasado se mantuvieron constantes. 
Cuadro 3. Constantes para la determinación de la concentración de sal para cada uno de los factores en estudio, tiempo (T), tipo de sal (S), tipo de envasado (E) y sus interacciones.

\begin{tabular}{|c|c|c|c|c|c|c|c|c|}
\hline & $\mathbf{T}$ & E & S & TxE & SxE & TxS & $\mathbf{R}^{2}$ & $\mathbf{R}^{2}$ ajustada \\
\hline \multicolumn{9}{|c|}{ Sal ahumada } \\
\hline$a_{w}$ & 0,10 & $-0,0005$ & 0,006 & $-0,00001$ & $-0,002$ & 0,00006 & 0,83 & 0,82 \\
\hline$Z^{\mathrm{NaCl}}$ & $-0,01$ & 0,02 & 0,02 & 0,00008 & $-0,002$ & $-0,0001$ & 0,88 & 0,87 \\
\hline$X^{\mathrm{NaCl}}$ & 0,10 & 0,04 & $-0,05$ & 0,00006 & $-0,002$ & $-0,0002$ & 0,96 & 0,95 \\
\hline \multicolumn{9}{|c|}{$\mathrm{NaCl}$} \\
\hline$a_{w}$ & 0,10 & $-0,003$ & & 0,000003 & & & 0,83 & 0,82 \\
\hline$Z^{\mathrm{NaCl}}$ & 0,02 & 0,002 & & 0,00005 & & & 0,81 & 0,80 \\
\hline $\mathrm{X}^{\mathrm{NaCl}}$ & 0,10 & 0,020 & & 0,00006 & & & 0,85 & 0,84 \\
\hline
\end{tabular}

La Figura 3 muestra los resultados de la RSM, la combinación de factores estableció que transcurridos 161 minutos se logran los mejores resultados del proceso de salado, al alcanzar un valor de actividad de agua de 0,95 y un contenido de sal de $0,042 \mathrm{~g} \mathrm{NaCl} / \mathrm{g}$ en la fase líquida, que corresponde a un valor de de $0,17 \mathrm{~g} \mathrm{de} \mathrm{NaCl} / \mathrm{g}$ en base seca al utilizar sal ahumada con envasado al vacío, Figura 4(A); en este tipo de salado se aporta el sabor característico a humo que está relacionado con una mayor aceptación sensorial (Obeng et al., 2018).

El modelo obtenido de la RSM mostró que el $66 \%$ de las combinaciones de los factores generan respuestas favorables para el objetivo de sal establecido por el Codex Alimentarius (CODEX, 2013), lo que garantiza la calidad y seguridad del producto. Al comparar el resultado de la optimización (sal ahumada) con el uso del $\mathrm{NaCl}$ se observó que el tiempo de salado fue de 129 minutos con un valor de actividad de agua de 0,95 y un contenido de sal óptimo de $0,056 \mathrm{~g} \mathrm{NaCl} / \mathrm{g}$ en la fase líquida, lo que corresponde a un valor de de 0,19 g de NaCl/g en base seca al utilizar el envasado al vacío con una deseabilidad de 0,73, Figura 4(B).

A

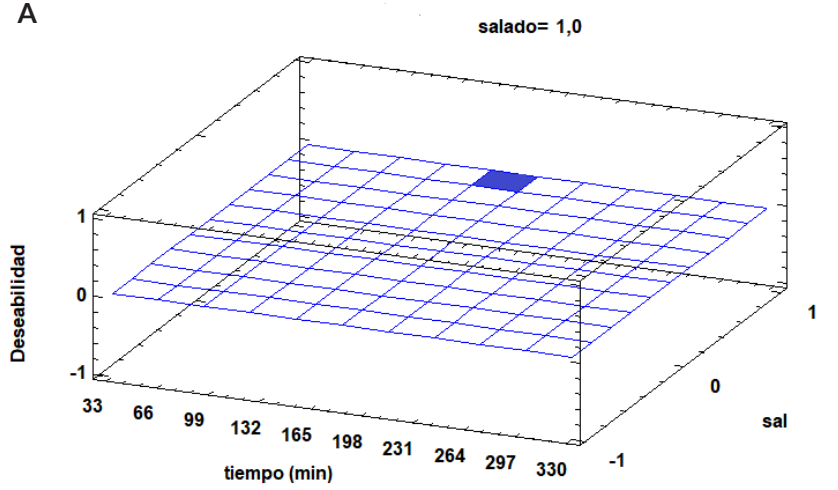

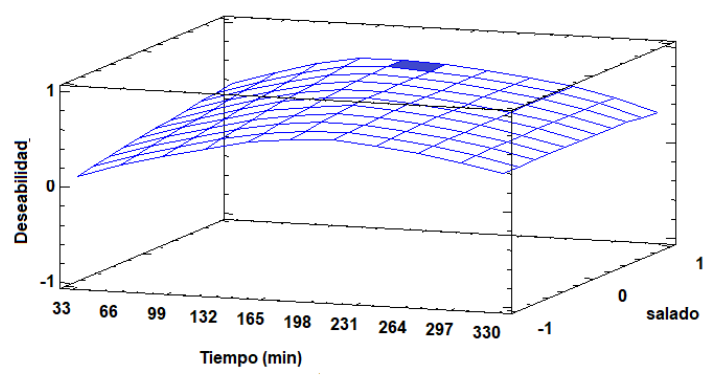

Figura 3. Superficie de respuesta del proceso de salado de filetes de tilapia. Óptimo: sal ahumada con en envasado al vacío (A). Optimización con $\mathrm{NaCl}$ con envasado al vacío (B).

Por tanto, la combinación de factores en cada uno de los modelos cumple con las normas descritas por Codex Alimentarius (CODEX, 2013), que establece para productos de pescado salado un contenido mínimo de sal que debe estar entre el 3 y 3,5 \% y máximo un $5 \%$ para proporcionar protección completa a temperaturas de refrigeración entre 3 y $10^{\circ} \mathrm{C}$, con lo cual el desarrollo del producto, según los niveles de sal encontrados, no afecta la salud del consumidor. 


\section{CONCLUSIONES}

El proceso de salado evaluado mostró un transporte pseudo-difusional en todos los tratamientos frente a las variaciones de masa. La concentración ideal para el salado de los filetes se obtuvo utilizando sal ahumada y envasado al vacío. De los factores evaluados el tiempo de salado evidenció la mayor influencia frente al tipo de sal y el envasado, las interacciones fueron menos importantes que los factores considerados individualmente.

\section{REFERENCIAS}

ASSOCIATION OF OFFICIAL ANALYTICAL CHEMISTS (AOAC). Official Methods of Analysis. Arlington (USA): 16, 1997.

BJØRNEVIK, MARIT; CARDINAL, MIREILLE; VALLET, JEAN-LUC; NICOLAISEN, OVE; ÖRN, GUDMUNDUR. Effect of salting and cold-smoking procedures on Atlantic salmon originating from pre-or post rigor filleted raw material. Based on the measurement of physiochemical characteristics. LWT, v. 91, 2018, p. 431-438. https://doi.org/10.1016/j.lwt.2018.01.047

BOULETIS, ACHILEAS; ARVANITOYANNIS, IOANNIS; HADJICHRISTODOULOU, CHRISTOS. Application of modified atmosphere packaging on aquacultured fish and fish products: A review. Critical reviews in food science and nutrition, v. 57, n. 11, 2017, p. 2263-2285. https://doi.org/10.1080/10408398.2013.862202

CAMERO-ESCOBAR, GUILLERMO; CALDERÓN-CALDERÓN, HUGO. Vigilancia tecnológica e inteligencia competitiva para la producción de tilapia roja (Oreochromis mossambicus) en el departamento del Huila, Colombia. Revista de Investigación, Desarrollo e Innovación, v. 9, n. 1, 2018, p. 19-31. https://doi.org/10.19053/20278306.v9.n1.2018.8504

CASALES, MARÍA; YEANNES, MARÍA. Mass Transfer Modeling During Marination of Anchovy Fillets in Ternary and Multicomponent Solutions. Journal of aquatic food product technology, v. 25, n. 5, 2016, p. 760-769. https://doi.org/10.1080/10498850.2014.924168

CHANG-CHENG, ZHAO; JONG-BANG, EUN. Influence of ultrasound application and $\mathrm{NaCl}$ concentrations on brining kinetics and textural properties of Chinese cabbage. Ultrasonics sonochemistry, v. 49, 2018, p. 137-144. https://doi.org/10.1016/j.ultsonch.2018.07.039

CHANG-CHENG, ZHAO; JONG-BANGI, EUN. Kinetic study of mass transfer in different parts of Chinese cabbage during brining. Journal of food process engineering, v. 41, n. 3, 2018, p. 2-7. https://doi.org/10.1111/jfpe.12666

ITALIA. CODEX ALIMENTARIUS. Standard for Smoked Fish, Smoke-Flavoured Fish and Smoke-Dried Fish. FAO/WHO. Rome (Italy): 2013

DEUMIER, FRANÇOIS. Pulsed-vacuum immersion of chicken meat and skin in acid solutions. Effects on mass transfers, colour and microbial quality. International Journal of Food Science and Technology, v. 39, 2004, p. 277-286. https://doi.org/10.1111/j.1365-621.2004.00782.x

ERKAN, NURAY. The effect of active and vacuum packaging on the quality of turkish traditional salted dried fish "çiroz". Food and Health, v. 3, n. 1, 2017, p. 29-35. https://doi.org/10.3153/JFHS17004

FOUAD, FATIMAEZZAHRA; TOURABI, AMINA; LAKHNATI, GHIZLANE. The innovation process impact on the new product performance: a case study. International Journal of Innovation Science, v. 10, n. 3, 2018, p. 385-412. https://doi.org/10.1108/IJIS-08-2017-0071

FUENTES, ANA; BARAT, JOSÉ; FERNÁNDEZ-SEGOVIA, ISABEL; SERRA, JUAN. Study of sea bass (Dicentrarchus labrax L.) salting process: kinetic and thermodynamic control. Food Control, v. 19, n. 8, 2008, p. 757-763. https://doi.org/10.1016/j.foodcont.2007.07.014 
GALVÃO-MARTINS, MAYARA; NUNES-CHADA, PAULO; DA SILVA-PENA, ROSINELSON. Application of pulsed-vacuum on the salt impregnation process of pirarucu fillet. Food Research International, v. 120, 2019, p. 407-414. https://doi.org/10.1016/j.foodres.2019.03.016

GARCIA, ANDRÉ; DE OLIVEIRA, CARLOS; KARIM, HANNER; SARY, CÉSAR; TODESCO, HUMBERTO; RIBEIRO, RICARDO. Genetic parameters for growth performance, fillet traits, and fat percentage of male Nile tilapia (Oreochromis niloticus). Journal of Applied Genetics, v. 58, n. 4, 2017, p. 527-533. https://doi.org/10.1007/s13353-017-0413-6

GARCÍA-BERNAL, ADRIANA; ARIZA, SHARON; FLÓREZ-RINCÓN, LAURA. Biocomercio: una práctica empresarial en el cultivo y exportación de la tilapia roja para mejorar el sector piscícola en Colombia, caso de estudio en el departamento del Huila 2005-2013 [Tesis Profesional en Finanzas y Comercio Internacional]. Bogotá (Colombia): Universidad de la Salle, Facultad de Ciencias Económicas y Sociales, 2016, 100 p.

GIRÓN, JOEL; GIL-SÁNCHEZ, LUÍS; GARCÍA-BREIJO, EDUARDO; PAGÁN, MARÍA; BARAT, JOSÉ; GRAU, RAÚL. Development of potentiometric equipment for the identification of altered dry-cured hams: A preliminary study. Meat Science, v. 106, 2015, p. 1-5. https://doi.org/10.1016/j.meatsci.2015.03.006

GUIMARÃES, BIANCA; NUNES DE SÁ, ANA; RAMOS, AMANDA; CASAÑAS, ANNA. Evaluation of texture and color of ready-to-eat food processing by ionizing radiation. Book of abstracts International Nuclear Atlantic Conference. Santos (Brazil): Associação Brasileira de Energia Nuclear, 2019, p. 2030-2037.

JIANG, QINGQING; NAKAZAWA,NAHO;HU, YAQIN; OSAKO, KAZUFUMI;OKAZAKI, EMIKO. Microstructural modification and its effect on the quality attributes of frozen-thawed bigeye tuna (Thunnus obesus) meat during salting. LWT, v. 100, 2019, p. 213-219. https://doi.org/10.1016/j.lwt.2018.10.070

LAUB-EKGREEN, MARIA; JESSEN, FLEMMING; MARTINEZ-LOPEZ, BRAIS. Mechanistic modelling of the coupled salt and water transport in herring during brining and curing. Journal of Food Engineering, v. 250, 2019, p 18-25. https://doi.org/10.1016/j.jfoodeng.2019.01.013

LÓPEZ-VALENCIA, YVETH. Estudio de la cinética de deshidratación osmótica para la conservación de lisa (Mugil cephalus) [Tesis Ingeniería Pesquera]. Arequipa (Perú): Universidad Nacional de San Agustín de Arequipa, Facultad de Ciencias Biológicas, 2017, 154 p.

MARIÑO-GARCÍA, JESSICA. Estudio de factibilidad de proyecto empresarial de producción y comercialización de mojarra roja con proyección en la ciudad de Bogotá [Tesis en Ingeniería Industrial]. Bogotá (Colombia): Universidad Católica de Colombia, Facultad de Ingeniería, Programa de Ingeniería Industrial, 2018, 160 p.

MARTINS, MAYARA; CHADA, PAULO; DA SILVA-PENA, ROSINELSON. Application of pulsed-vacuum on the salt impregnation process of pirarucu fillet. Food Research International, v. 120, n. 1, 2019, p. 407-414. https://doi.org/10.1016/j.foodres.2019.03.016

MONTEIRO, MARIA; MÁRSICO, ELIANE; ROSENTHAL, AMAURI; CONTE-JUNIOR, CARLOS. Synergistic effect of ultraviolet radiation and high hydrostatic pressure on texture, color and oxidative stability of refrigerated tilapia fillets. Journal of the Science of Food and Agriculture, v. 99, n. 9, 2019, p. 4474-4481. https://doi.org/10.1002/jsfa.9685

OBENG, EUGENE; ONGKUDON, CLARENCE; BUDIMAN, CAHYO; MAAS, RUTH; JOSE, JOACHIM. An optimal blend of single auto displayed cellulases for cellulose saccharification - a proof of concept. Journal of Chemical Technology and Biotechnology, v. 93, n. 9, 2018, p. 2719-2728.

https://doi.org/10.1002/jctb.5628

OLIVEIRA-ALCÂNTARA, LYNDERVAN; DE OLIVEIRA, MARIA; RABELO-SOUSA, JULIANA; CERQUEIRA, MIGUEL; COELHO-SILVA, ANDRÉ; MOREIRA-SOUZA, MEN DE SÁ. Wettability of edible coatings on Nile tilapia fillets (Oreochromis niloticus). Journal of Food Engineering, v. 247, 2019, p.152-159.

https://doi.org/10.1016/j.jfoodeng.2018.11.026

PARRADO-SANABRIA, YINET. Historia de la Acuicultura en Colombia. Revista Aquatic, v. 37, 2012, p. 60-77. 
PETIT, GAËLLE; JURY, VANESSA; DE LAMBALLERIE, MARIE; DURANTON, FRÉDÉRIQUE; POTTIER, LAURENCE; MARTIN, JEAN-LUC. Salt Intake from Processed Meat Products: Benefits, Risks and Evolving Practices. Comprehensive Reviews in Food Science and Food Safety, 2019, p. 1-21. https://doi.org/10.1111/1541-4337.12478

RIZO, ARANTXA; FUENTES-LOPEZ, ANA; FERNÁNDEZ-SEGOVIA, ISABEL; BARAT, JOSÉ. Development of a novel smoke-flavoured trout product: An approach to sodium reduction and shelf life assessment. Journal of Food Engineering, v. 211, 2017, p. 22-29. https://doi.org/10.1016/j.jfoodeng.2017.04.031

RIZO, ARANTA; FUENTES, ANA; BARAT, JOSÉ; FERNÁNDEZ-SEGOVIA, ISABEL. Development of a novel smoke-flavoured salmon product by sodium replacement using water vapour permeable bags. Journal of the Science of Food and Agriculture, v. 98, n.7, 2017, p. 2721-2728. https://doi.org/10.1002/jsfa.8767

RODRÍGUEZ-ORTIGOZA, ANGÉLICA. Probióticos en la producción piscícola[Tesis especialista en Biotecnología Agraria]. Neiva (Colombia): Universidad Nacional Abierta y a Distancia, Escuela de Ciencias Agrarias, Pecuarias y del Ambiente, 2017, 35 p.

TIRADO-ARMESTO, DIEGO-FELIPE; ACEVEDO-CORREA, DIOFANOR; MONTERO-CASTILLO, PIEDADMARGARITA. Secado de arenca (Triportheus magdalenae): influencia del salado en pila seca y húmeda. Biotecnología en el Sector Agropecuario y Agroindustrial, v. 14, n. 2, 2016, p. 57-64. https://doi.org/10.18684/BSAA(14)57-64. 